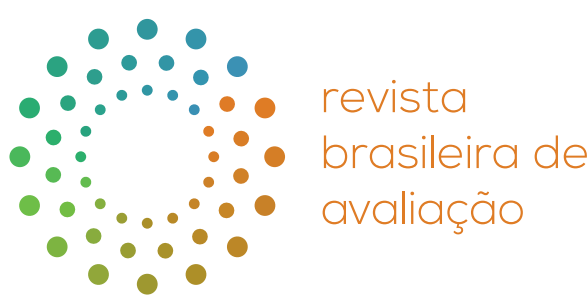

\section{Entrevista con la chilena Alejandra Faúndez: "En el caso de América Latina, el sofisticar la evaluación no tiene que ver con sofisticar el método. Tiene que ver con integrar la complejidad de esta región al análisis, que son caminos distintos"}

\author{
"In the case of Latin America, the sophistication of the evaluation \\ does not have to do with sophistication of the method. It has to \\ do with integrating the complexity of this region into the analysis, \\ which are different paths." An interview with Chilean Alejandra \\ Faúndez
}

\author{
Carolina Imura' ${ }^{1}$ [branca] (D, Marcos Assis ${ }^{1}$ [branco] \\ ${ }^{1}$ Fundação João Pinheiro, Belo Horizonte, MG, Brasil
}

CÓMO CITAR: Imura, Carolina, \& Assis, Marcos (2021). Entrevista con la chilena Alejandra Faúndez: “En el caso de América Latina, el sofisticar la evaluación no tiene que ver con sofisticar el método. Tiene que ver con integrar la complejidad de esta región al análisis, que son caminos distintos". Revista Brasileira de Avaliação, 10(2), e101621. https://doi.org/10.4322/rbaval202110016

\section{Resumen}

Las crisis de algún modo guardan una semilla, un germen de cosas nuevas que uno puede volver a revisar, a reinventar, a recrear. Entonces, en ese sentido, creo que fue un momento muy crítico, muy caótico también. O sea, había muchas pugnas de poderes, muchos intereses en juego, muchas cosas que derrumbar definitivamente. Pero también creo que había mucha esperanza. Las crisis siempre albergan esas dos tensiones. O sea, de un lado tanto dolor, tanta tragedia y, por otro lado, también tanta esperanza de que se pueden hacer mundos diferentes. Entonces creo que la crisis, en ese momento (de la dictadura en Chile) fue cuando a mí me encontró la evaluación, porque yo creo que la evaluación me encontró a mí más que yo a ella.

Palabras clave: Evaluación. Enfoque de género. Equidad. Latinoamérica.
RBAVAL supports efforts related to the visibility of African descent authors in scientific production. Thus, our publications request the self-declaration of color/ethnicity of the authors to make such information visible in the journal.

Recibido: Agosto 25, 2021

Aceptado: Agosto 27, 2021

*Autor correspondiente:

Carolina Imura

E-mail: carolimura@gmail.com

\section{(cc) BY}

Este é um artigo publicado em acesso aberto (Open Access) sob a licença

Creative Commons Attribution, que permite uso, distribuição e reprodução em qualquer meio, sem restrições desde que o trabalho original seja corretamente citado. 
Alejandra, tienes una maestría en gestión de políticas públicas de la Universidad de Chile, eres investigadora en el área de género y políticas sociales, fuiste coordinadora de docencia y extensión en FLACSO. También eres evaluadora de organismos públicos nacionales e internacionales como UNFPA, UNICEF, PNUD, CEPAL, BID, OPS / OMS, SEGIB y AECI en temas de políticas públicas, indicadores y planificación sociales con enfoque participativo y de género. Has publicado extensamente y trabajado para organismos internacionales y para el Comité Académico de la Red de Evaluación, Seguimiento y Monitoreo de América Latina y el Caribe (RELAC) desde su fundación, así como fundadora de varias redes en la región ¿Cómo ingresaste al campo de la evaluación centrada en el género?

Inicié en los temas de género muy temprano, desde cuando ingresé en la universidad para estudiar trabajo social y desde allí me vinculé laboralmente con FLACSO Chile, donde estábamos haciendo investigaciones sobre los temas de género. Creo que esta vinculación académica vino de una trayectoria dentro del movimiento de mujeres desde adolescente, después participando en la Federación de estudiantes de la Universidad de Chile, experiencias en las que siempre estuve vinculada a los derechos de las mujeres, mi tesis de grado incluso fue sobre las mujeres estudiantes en la Universidad de Chile. Desde ahí, fue casi natural que, ya trabajando en FLACSO, me involucraría en estudios desde una perspectiva de igualdad de género.

Cuando llegué ahí, se estaba desarrollando un estudio comparativo que fue muy conocido en la región, que se llamó "Mujeres Latinoamericanas en cifras". Eso implicó para mí comenzar a introducirme muy fuertemente en los temas de indicadores de género y a familiarizarme con la información de los distintos institutos de estadística de los países de la región. Más tarde, me empecé a especializar mucho en los temas estadísticos, y desde allí fui derivando a los temas de evaluación. Eso pasó en un momento en que los límites entre la investigación y la evaluación eran todavía muy difusos, muy porosos, no estaba claro si la intención del estudio era atribuir un juicio evaluativo. Fue un momento de mucha ebullición de saberes y de discusiones súper interesantes en el marco del desarrollo y metodológicas también.

Empezaba en ese momento la democracia en mi país, a fines de los años 80 más o menos. Era un momento histórico muy creativo en que se nos demandaba -a los que estábamos en el plano de la investigación o de la evaluación- la construcción de una nueva institucionalidad, estábamos reinventándonos todo, digamos. Había una ebullición de muchos saberes y también, al mismo tiempo, una crítica muy profunda a los enfoques más tradicionales de la academia lo que permitió muchas rupturas y muchos comienzos también, en mi caso, un comienzo más académico de vinculación con el enfoque de género que ya me acompañaba desde la militancia juvenil.

Las crisis de algún modo guardan una semilla, un germen de cosas nuevas que uno puede volver a revisar, a reinventar, a recrear. Entonces, en ese sentido, creo que fue un momento muy crítico, muy caótico también. O sea, había muchas pugnas de poderes, muchos intereses en juego, muchas cosas que derrumbar definitivamente. Pero también creo que había mucha esperanza. Las crisis siempre albergan esas dos tensiones. O sea, de un lado tanto dolor, tanta tragedia y, por otro lado, también tanta esperanza de que se pueden hacer mundos diferentes. Entonces creo que la crisis, en ese momento (de la dictadura en Chile) fue cuando a mí me encontró la evaluación, porque yo creo que la evaluación me encontró a mí más que yo a ella.

\section{¿Cómo surgió el campo de la evaluación sensible al género en la región?}

La pregunta me hizo recordar de un profesor de estadística que dijo algo muy definitivo, pero también gracioso sobre esto y que en mí marcó mi vida profesional: Para él, "los promedios eran como los trajes de baño, porque, en realidad, ocultan lo importante". La frase me marcó porque prácticamente sólo había trabajado con promedios: de mi país, de una región, de las mujeres, de los hombres, etc. Y buscando descubrir lo que estos promedios ocultaban, me metí en los temas de la desagregación de datos, me di cuenta de que muchas de las informaciones 
- Entrevista con la chilena Alejandra Faúndez: "En el caso de América Latina, el sofisticar la evaluación no tiene que ver con sofisticar el método. Tiene que ver con integrar la complejidad de esta región al análisis, que son caminos distintos"

que manejábamos en el campo de la evaluación no tenían esa mirada desagregada, y que en la evaluación seguíamos mirando, incluso cualitativamente, los discursos y narrativas que reproducían ciertos patrones, ideas y clichés de la cultura hegemónica y dominante. Creo que esa mirada tan sesgada fue la que, de alguna manera, me llevó a cuestionar que algo estaba faltando, había una pieza de este rompecabezas que no me estaba siendo coherente con lo que miraba.

Junto con eso, también ocurrió que había un movimiento de muchas investigadoras y evaluadoras, en distintos lugares del mundo, también haciéndose estas preguntas. En algún momento de esta historia laboral, no recuerdo exactamente de qué manera, me topé con una investigadora chilena que vive en España, en Barcelona, hace muchos años, que es Cristina Carrasco, quien trabajó durante muchos años de su vida el tema de la cuantificación/ monetización del trabajo doméstico. Ella me decía: “el trabajo no puede ser sólo lo que remuneramos, porque hay todo un conjunto de actividades que se desarrollan en el espacio doméstico que yo estoy intentando hace años valorar económicamente, y eso no lo vemos, incluso eso cambia el concepto mismo del trabajo humano".

Cuando tuve ese primer remezón conceptual respecto a lo que entendemos por trabajo, yo dije: "Bueno, pero entonces hay que cuestionarse otras cosas también". Esos debates derivaron en muchos estudios en la región, en las encuestas de uso del tiempo, en las cuentas satélites en los institutos de estadísticas, en la conceptualización y políticas de Cuidados, en el análisis de la economía del cuidado, en el aporte no remunerado al PIB, etc. Entonces, me cuestioné: a lo mejor, el mismo concepto de género que estamos trabajando es o está restringido y no lo hemos mirado críticamente. A lo mejor el concepto de salud y de educación no lo hemos mirado críticamente. O sea, me empecé a cuestionar muchas otras cosas sobre la base conceptual de lo que estaba haciendo. Y también dije: "Bueno y el concepto de evaluación también pues", o sea desde qué paradigma nos ponemos hacer juicios evaluativos, desde qué lugar, desde qué marco conceptual, desde qué lectura de la realidad. Entonces desde allí empezamos a dialogar con bastantes otras colegas fabulosas que estaban en estos temas, haciéndose las mismas preguntas.

Apareció en algún momento nuestra colega Ada Ocampo. Ella estaba trabajando en el FIDA en ese momento como jefa o evaluadora de ese equipo en Roma. Ella estaba preguntándose respecto de las evaluaciones de proyectos que se hacían en este programa en América Latina. Empezamos -sin conocernos siquiera- un cuestionamiento más metodológico respecto de cuáles son las dimensiones que uno mira en una evaluación, qué pasa con el tema de los roles, que tan presentes están, qué pasa con la institucionalidad pública y el uso del tiempo de las mujeres para el desarrollo de proyectos de este tipo. Además, yo estaba trabajando en una institución pública y formaba parte de un equipo grande con indígenas y no indígenas, y todo esto lo digo porque voy a derivar a eso también después del tema de género. Y ahí estaba a cargo de la planificación y la evaluación del programa en Chile.

Y yo seguía cuestionando: qué es lo que registro, qué no registro, qué nivel de desagregación tienen mis datos, cómo los muestro, qué digo, cómo los interpreto, qué piensan las Mapuche, qué piensan las Aimaras, qué piensan las Rapanui de estos datos, cómo los leen ellas? O sea, empecé a mirar de otra manera la información que uno tradicionalmente llama "objetiva".

Entonces el tema de género en evaluación para mí viene de nuevo con esta marca más biográfica, desde el activismo y, al mismo tiempo, desde esta crítica más académica, desde una lógica más constructiva de cómo entre todos y todas vamos generando nuevos saberes. Creo que esos tres ingredientes también se dieron en torno a los temas de género en la evaluación.

En un plan más académico, cuando volví a FLACSO en el 2005, me tocó hacerme cargo de la coordinación académica y la extensión de la Facultad en Chile. Esta era la primera vez después de la dictadura que volvíamos a abrir el espacio académico en la institución, un espacio no investigativo, sino docente. Y entonces, se me ocurrió -con un grupo de colegas- inventar un diplomado en evaluación y que terminó con una mirada latinoamericanista muy interesante, amplio y diverso, recogiendo mucha de la expertise de la región. En ese diplomado, además, 
- Entrevista con la chilena Alejandra Faúndez: "En el caso de América Latina, el sofisticar la evaluación no tiene que ver con sofisticar el método. Tiene que ver con integrar la complejidad de esta región al análisis, que son caminos distintos"

teníamos un curso específico de género en evaluación, que hicimos muy a la medida con varias colegas, con Elizabeth Guerrero, Soledad Quiroz y varias otras, después se fueron sumando a esta reflexión muchas más: Belén Sanz, al asumir como Jefa de evaluación de la recién creada ONU Mujeres y que actualmente es la representante de la ONU mujeres en México, con Laura González, que venía de la AECID y fue jefa de evaluación regional de la ONU mujeres, con Carmen Colazo de Paraguay, Marcia Paterno de Brasil, Silvia Salinas de Bolivia, Gloria Vela y Fabiola Amariles de Colombia, y también las europeas que estaban haciendo lo propio como María Bustelo y Julia Espinosa En definitiva, con muchas mujeres -que estábamos repartidas en distintos países estábamos con estas mismas inquietudes. Fue, yo creo, un momento de incorporar el género, muy desde una mirada más intuitiva al inicio, en que fuimos haciendo un ejercicio de prueba y error, encontrándonos e intercambiando hallazgos.

Entonces creo que, esa experiencia de diplomado y después todo el trabajo que hicimos con el Grupo de Género y Derechos Humanos en la RELAC y con ONU Mujeres durante bastantes años, fortaleciendo capacidades en evaluación con enfoque de género, fue de alguna manera haciendo puentes entre este mundo académico, entre la investigación, entre la evaluación, entre el análisis además de los fenómenos que afectaban a las mujeres a nivel de la región en América Latina y en el mundo, de manera a ir construyendo un acervo conceptual, metodológico, que iba dando cuenta de todas esas miles de mujeres que andaban por ahí, digamos, con los mismos objetivos e inquietudes y que queríamos construir una voz propia desde el Sur global.

\section{Hablaste de comprender, construir y definir conceptos. ¿Cuál es el concepto de género en la evaluación y qué similitudes y diferencias hay entre este concepto $y$, por ejemplo, la perspectiva de evaluación y equidad?}

Lo primero que tenemos que reconocer y visibilizar es que el concepto de género en Latinoamérica en particular, pero también en otras del mundo, ha tenido una evolución. Hoy día no hablamos del mismo género que hace 10 años atrás o que hace 20 años atrás. ¿Y qué quiero decir con esto y cuál es su relación con los temas de la evaluación? Yo creo que el género se incluyó en la evaluación en un momento en que necesitábamos contabilizar personas de manera diferenciada. Entonces muchos de los esfuerzos evaluativos que se hacían tenían que ver precisamente con buscar los datos de las personas "impactadas" o "tocadas" por un proyecto en tanto eran mujeres u hombres. Durante bastante tiempo, lo que se hizo fue intentar contar con estadísticas desagregadas y ahí acababa todo.

Después de eso, después que pudimos ver los datos y analizarlos, nos dimos cuenta de que eso no era suficiente, de que cuando hablamos de género estábamos hablando de algo más. Y ese algo más, para muchas de las investigadoras y metodólogas de América Latina, se tradujo en hacer análisis sobre la diferenciación de roles de hombres y mujeres en ciertas temáticas o proyectos que evaluábamos, como también sobre el uso del tiempo vinculado a esos roles y la situación, condición y posición de las mujeres en los proyectos. Creo que muchas de las evaluaciones lo que mostraron efectivamente fue eso: aunque un proyecto tuviera un mismo objetivo, una misma cantidad de recursos, la misma cantidad de población femenina y masculina, etcétera, los proyectos impactaban a esa población de manera diferenciada.

Además, Marco Segone tuvo un rol fundamental cuando cuestionó el concepto mismo de desarrollo y de evaluación al decir: sabes que llegó el momento en que hay que entender que "el desarrollo le sucede a las personas en cualquier tipo de programa y cualquier tipo de proyecto". Eso significó un hito en la discusión de estos temas y es que entonces las que veníamos haciendo evaluaciones con enfoque de género dijimos: "Sí. Podríamos evaluar también obras públicas con enfoque de género. También podríamos evaluar el uso del agua con enfoque de género, podríamos...Claro." Porque si el desarrollo le sucede a las personas, entonces en cualquier cosa que se haga la perspectiva del desarrollo está impactando a mujeres y hombres de manera diferenciada. Y así fue como también muchas investigadoras y evaluadoras empezamon a incursionar en ámbitos no tradicionales al análisis de género. 
- Entrevista con la chilena Alejandra Faúndez: "En el caso de América Latina, el sofisticar la evaluación no tiene que ver con sofisticar el método. Tiene que ver

De ahí salió que en muchos ámbitos del desarrollo empezaron a formarse especialistas en enfoque de género. Y en la evaluación también.

En este contexto, muchas de nosotras empezamos a intentar perfeccionar el método, a discutir las metodologías cuanti, discutir los enfoques metodológicos y los enfoques tradicionales de evaluación de impacto o de resultado, así como las salvaguardas éticas con las personas entrevistadas. Y eso nos llevó al tema de la participación en la evaluación, porque siempre decimos que tenemos programas o que estamos evaluando participativamente un programa $y$, sin embargo, lo único que hacemos es consultar. O sea, las personas que son los/as destinatarios/as, digamos, de ese proyecto, son consultados, pero raramente definen los indicadores de la evaluación, raramente definen las preguntas de la evaluación, raramente opinan sobre los hallazgos de la evaluación.

Empezamos también a ampliar el punto de vista binario de mujeres y hombres y a incorporar otros actores a la escena, como las mujeres indígenas, que no están señaladas en el promedio del universo de mujeres y a las mujeres negras (como se autodenominan en Brasil), indicando, por ejemplo, que una mujer afrodescendiente embarazada beneficiaria de un programa de prevención de embarazo en un quilombo de Brasil no es igual a una mujer afrodescendiente que vive en el Bronx Nueva York. Creo que todas esas discusiones que se estaban dando en el campo del género, de la diversidad y del ejercicio de derechos, también fueron impactando a la evaluación.

Volviendo al tema de cómo se fue manejando el concepto de género, el de igualdad y equidad, también va teniendo un desarrollo en paralelo en las propias ciencias sociales, en algún momento hubo confusiones y caricaturas sobre el tema de la igualdad de género, suponiendo -equivocadamente- que las mujeres queríamos "parecernos" a los hombres, ique aberración más grande! Y sin embargo esas discusiones se daban en aquel entonces, desconociendo todas las luchas y esfuerzos por el ejercicio igualitario de derechos. También nos dimos cuenta, en el trabajo de evaluación con organismos internacionales, que había enfoques que funcionaban de manera paralela. O se aplicaba el enfoque de género o el enfoque de derechos humanos y no necesariamente ellos dialogaban entre sí. Entonces nos sentamos con un grupo grande de colegas a decir: "Bueno, ¿qué hacemos con estos dos enfoques? ¿qué tenemos que integrar? ¿cómo se hace?" Y nos respondimos: "tenemos que ver las interfaces de articulación entre todo esto, tenemos que tender puentes".

Y justo por las cosas un poco mágicas que también ocurren en la vida, nos pidieron hacer una consultoría de integración del enfoque de género y derechos humanos para cuatro agencias regionales de Naciones Unidas en América Latina. Nos dedicamos a investigar qué hacía cada agencia, los indicadores y metodologías que utilizaban y entrevistamos a distintos evaluadores, hasta que logramos publicar un guía que se llama "Ampliando la mirada", que resume esa mirada crítica y las tensiones que hay respecto de los marcos conceptuales que manejábamos en esos momentos en la región, pero desde una óptica propositiva.

Al fin y al cabo, creo que nuestro primer esfuerzo para conceptualizar fue tejer puentes entre muchas teorías, evidencias, tensiones y nudos estratégicos que caracterizan la desigualdad en América Latina en particular.

\section{¿Ya has trabajado en una evaluación con enfoque de género pasando por todas las etapas (planificación, ejecución y comunicación de resultados)? ¿Qué podemos hacer para transformar las evaluaciones y sus instrumentos más comunes en herramientas que consideren la perspectiva de género?}

Desde EVALGender, la primera declaración que hicimos de evaluación con enfoque de género fue hablar del Gender+, un concepto en inglés. Eso significaba mirar precisamente el tema de la interseccionalidad en el caso de América Latina, o sea, no solo mirar la dimensión de género desde una óptica binaria, sino también incorporar otras desigualdades u otras opresiones que se vinculan, que se intersectan y que, además, resultan en situaciones de desigualdad diferentes si las miráramos sólo desde hombres y mujeres. 
Yo diría que en el proceso de planificación de la evaluación nos tocó cuestionar la participación de los involucrados en la evaluación. Si estamos haciendo una evaluación en una zona geográfica donde hay colectivos organizados que representan a estas diferentes formas de vida, hay que invitar a esos colectivos como grupo, no solamente como personas destinatarias del proyecto. Y eso es sumamente desafiante para todas las instituciones, o sea, todos estos temas de la complejidad no solo son una complejidad teórica, sino también práctica que pasa por quién financia la participación de esas personas, y como debe ser esa participación. Creo que siempre hay que considerar estos elementos en la planificación, sobre todo cuando uno quiere efectivamente hacer un trabajo participativo de verdad profundo.

Después, en el trabajo de campo también hay otras complejidades que tienen que ver con que muchos colectivos y comunidades están sobre encuestadas. Eso también va produciendo otros sesgos y otros problemas en la evaluación porque los colectivos empiezan a aprender qué es lo que el otro quiere oír. Empiezan a decirte las mismas cosas a ti que las que dijeron en otra evaluación que era de otra cosa nada que ver. Yo creo que la respuesta a esa complejidad es cómo vamos simplificando también las herramientas, los contactos, los encuentros y a la vez ampliando las muestras y el perfil de las personas a las que entrevistamos o encuestamos, en fin.

También es importante en el plan del trabajo de campo la presencialidad del evaluador, que no tiene que ver solo con estar ahí presente en el momento de la evaluación, sino también conocer y comprender los contextos y los entornos en que se dan determinados proyectos, 0 sea, hacer un trabajo de involucramiento con la realidad, con la situación o las cosas concretas que uno va a evaluar.

Creo que hay que superar urgentemente la concepción tradicional que tenemos del trabajo de campo como colector de información que después se pone en una canastita y se analiza. $\mathrm{Y}$, por lo tanto, ahí también es muy importante el tema de la lengua, de las costumbres, de la cultura, de los conocimientos del país, de la región, es decir, tener siempre a alguien en el equipo que sea un local, que pueda entender entonces desde una perspectiva más histórica ciertos procesos, que hable la lengua de las personas del proyecto que se está evaluando.

Por fin, respecto de los resultados, yo creo que, considerando los aportes del enfoque de género en una evaluación, los resultados pueden ser analizados no sólo en torno a los objetivos del proyecto y a los indicadores o metas propuestos, sino también a partir de una matriz de análisis de esos resultados en función de la superación de la desigualdad. Los resultados que uno obtenga de la evaluación tienen que dialogar con las grandes transformaciones que se aspiran desde la igualdad de género, los derechos de las mujeres y el empoderamiento de mujeres y niñas.

Les voy a poner un ejemplo que para mí es ilustrativo de esto que digo. Hace muchos años atrás, como en el 2009, hicimos una evaluación de la política de igualdad de género en una gran ciudad de América Latina. Entonces evaluamos todo lo que se había hecho en los temas de género en distintas áreas del desarrollo y una de esas era violencia. Uno de los programas de violencia era el metro con carros solo para mujeres, y carros mixtos y también los buses rosa, en que van solo mujeres y niños. Entonces, cuando nos tocó entrevistar a una autoridad de Transporte, toda su narrativa obedecía a entender que ellos habían tenido muy buenos resultados con esto de la instalación de la movilidad separada para mujeres a través de este mecanismo separatista, lo cual se podía ver incluso en su propio presupuesto. Nos decía: "Mira, en el presupuesto del ministerio he incrementado todos los años los buses rosa. Eso de alguna manera les da mayor seguridad a las mujeres para poder habitar la ciudad y trasladarse". Después yo le pregunté: "Bueno, ¿y usted cree que ha disminuido entonces la violencia de género en el transporte público?". Y él me dice: "Bueno, yo creo que sí ha disminuido, pero eso no lo podemos decir porque si no nos van a bajar el presupuesto".

Entonces en los temas de género es clave que uno tenga muy, muy clara y definida, la ruta de esos cambios, de cómo quiere aproximarse a esas transformaciones, porque lo otro son métodos, mecanismos que tienen que estar en la dirección de los cambios. Entonces podría pasarme la vida pensando en que soy exitosa si tengo $20,30,40,50,1000$ carros rosa en la 
- Entrevista con la chilena Alejandra Faúndez: "En el caso de América Latina, el sofisticar la evaluación no tiene que ver con sofisticar el método. Tiene que ver con integrar la complejidad de esta región al análisis, que son caminos distintos"

ciudad, pero eso no me dice nada respecto de si superé la violencia de género en el transporte público. Es por eso que me parece que, en el plano de la comunicación de resultados, es muy importante tener a la vista cuáles son las transformaciones más estructurales que se precisan en el campo del género, y ellas están muy estudiadas, son parte de los 4 nudos estructurales de la Agenda regional de Género en América Latina.

Si no entendemos estas pequeñas transformaciones que van produciendo los proyectos en función de grandes transformaciones estructurales, vamos a seguir dando vueltas en la rueda en el tema de género y vamos a seguir reproduciendo estas desigualdades de maneras más sutiles, pero en el fondo sin soluciones reales.

\section{En tus experiencias, ¿cómo los gobiernos y sus ministerios y secretarías ven las evaluaciones con enfoque de género? ¿Hay presupuestos para estas evaluaciones?}

Yo tengo dos opiniones respecto de ese tema. Una que tiene que ver con la opacidad, diría yo, que tiene el mundo de la consultoría en evaluación en América Latina. Como evaluadora nunca alcanzo a comprender por qué un gobierno destina 10.000 dólares y otros 100.000 a los estudios evaluativos de proyectos que son similares en su alcance y magnitud. No necesariamente los costos están asociados al tipo de complejidad de la evaluación ni al tiempo que se le dedica, o sea, no hay una relación tan obvia ni tan automática en estas cosas. Entonces creo que ahí hay un primer desafío que avanzar: estandarizar un poco más y hacer más transparente también el sistema de contrataciones de evaluación en la región.

El segundo tema respecto a los recursos tiene que ver con la cantidad de recursos que se destina a la evaluación, a mí me da la impresión de que ha ido a la baja. En un momento hace unos años atrás, fue muy fuerte la imagen de que todos los países estaban instalando sistemas nacionales de evaluación y que estaban aportando recursos, aunque fueran mínimos, pero de alguna manera se estaba consolidando una lógica de institucionalización de los sistemas de evaluación. Pero creo que eso ha sido muy diferente entre países, pues siempre insisto que en América Latina las instituciones van y vienen, a veces desaparecen y en un par de años vuelven a aparecer. O sea, no necesariamente hay un acumulado que vaya en una dirección ascendente.

Hemos notado que muchas de las evaluaciones ahora tienen que ser más rápidas, más cortas en tiempo, más profundas, más complejas, pero con menos financiamiento. Yo creo que esa ecuación es fatal para el proceso de evaluación, para dar buenos resultados en calidad. A eso se suma que los gobiernos han destinado pocos recursos a la formación de sus comunidades de evaluadores. Pienso que no hay presupuesto suficiente, tampoco capacidades construidas para invertir en evaluaciones en general y es igual con la inclusión del enfoque de género, quieres que tenga todo, pero no inviertes nada

\section{A menudo, los dibujos evaluativos vienen listos en los términos de referencia. ¿Es posible influenciar el diseño de estas evaluaciones? ¿Cómo?}

Sí, es posible, pero hay elementos externos que influyen mucho más que los propios evaluadores. Una experiencia muy ilustrativa en esto fue una evaluación de impacto que hicimos para un organismo multilateral en Honduras sobre pueblos indígenas y afros. La evaluación empezó el año 2008 y todavía no habíamos presentado siquiera los resultados cuando hubo el golpe de Estado en Honduras. Eso demuestra que uno puede cumplir con todos los pasos rigurosos de una evaluación y, de repente, te sorprende un evento político como ese que nunca fue considerado y ya no se puede ni seguir el proceso ni presentar los resultados ni nada. En estos casos, algunas preguntas se plantean, como:¿Qué sentido tiene el cumplir todos los pasos si la evaluación está descontextualizada? ¿si no se es capaz de considerar todos los elementos del entorno político o medioambiental y tener capacidad de adaptación a eso? Si hubiéramos previsto que, en algún momento no íbamos a presentar los resultados a tiempo, entonces deberíamos haber diseñado metodologías distintas o haber 
- Entrevista con la chilena Alejandra Faúndez: "En el caso de América Latina, el sofisticar la evaluación no tiene que ver con sofisticar el método. Tiene que ver

diseñado mecanismos para tener impacto en la comunidad y en la propia institucionalidad pública a cargo

En esa misma evaluación de impacto nos sucedió que -como era casi un censo de pueblos indígenas-, fuimos a entrevistar a una comunidad que quedaba en una sierra, en una montaña que queda en la altura. Y cuando íbamos camino hacia allá nos encontramos con un temporal muy pero muy fuerte, un mini-huracán en esa zona y no podíamos subir. Así que nos quedamos albergados en el local de abajo de la comunidad, antes de subir la montaña. Y ahí descubrimos un señor que era cura de esa comunidad y que, en un momento de la conversación, nos dice: "No, pero no se queden aquí esperando sin hacer nada. Yo les puedo contestar todas las preguntas de los de arriba. No tienen que estar aquí toda la semana esperando que amaine la tormenta". Yo recuerdo que le decía: "No, gracias, es que esto tiene que ser súper riguroso porque tenemos que saber exactamente si hubo nacimientos, qué pasó con las familias, etc.". El cura insistía: "pero yo he bautizado a los niños así que yo sé los que nacieron, los que murieron, todo...", "Yo puedo incluso opinar por ellos, sé lo que piensan" (risas). Le dije: "No, gracias, vamos a esperar, lo vamos a hacer nosotros". Nos decía: "Bueno, pero ustedes están, en realidad, malgastando los recursos y su tiempo porque eso yo lo hago siempre. O sea, cada vez que vienen los del INE o del gobierno aquí, yo les informo todo lo que pasa arriba y no necesitan subir porque es muy peligroso". Esto que puede parecer fuerte o tragicómico es una realidad en muchos de nuestros países y hay que decirlo, hay que hablarlo para superarlo. Los $\mathrm{TdR}$ dicen algo, pero en la práctica evaluativa pueden suceder muchas otras cosas, algunas insospechadas y hay que dar cuenta de esas experiencias y aprendizajes.

A eso se suma que, cuando entra un nuevo gobierno muchos de los programas cambian y cambian tan radicalmente que se anula todo lo que se hizo anteriormente, de modo que se parte del cero mil veces. A mí me ha tocado evaluar programas del mismo tema en el mismo país como si nada hubiera existido antes.

Desde el punto de vista de la comunidad de evaluadores, deberíamos ir generando un estándar para salvaguardar la información, la memoria de los programas, cuando hay cambios de administración. Los diseños evaluativos pueden estar muy formateados, pero ya sabemos que siempre hay cosas que cambian, que cuestionan o que complejizan todo el proceso, hay que estar muy atentos a estas señales.

En definitiva, en clave de Boaventura de Souza, hay que prestar mucha atención a las emergencias (aquellos hitos, actores o situaciones inesperadas que emergen) y a las ausencias (todo lo que no incluiste en tu plan, lo que no es o no era visible).

\section{¿Cómo piensa sobre las perspectivas futuras del campo? ¿Qué desafíos debemos enfrentar para desarrollar el campo de la evaluación en América Latina?}

Por una parte, respecto de las agencias dedicadas o especializadas en evaluación, yo creo que ahí hay tres cosas que me parecen que son claves en este periodo que viene: Incrementar o al menos mantener los recursos, no solo los recursos económicos, sino también fortalecer sus propias capacidades como contraparte para diligenciar, diseñar y trabajar con los/as evaluadores externos. Y eso yo creo que es algo que se ha debilitado en estos últimos años. Hubo muchos diplomados en la región, hubo postgrados, maestrías y se fue instalando la idea de la necesidad de profesionalizar cada vez más y eso yo siento que hoy se ha estancado.

Otra cosa tiene que ver con las interrelaciones con la comunidad de evaluadores. A mí me parece, y eso también lo digo desde la comunidad de evaluadores, que es un riesgo el perder esas interfaces de conexión entre lo público y la comunidad de evaluadores independientes y externos. Porque ahí se arma una fisura que es muy artificial, porque nosotros vamos a seguir siempre evaluando. Van a haber periodos de tiempo en que esas distancias van a ser tan abismales que no van a tener posibilidades de mirar los programas y todo de una manera más amplia, articulada y compleja, y sobre todo se va perdiendo la capacidad de los organismos públicos de rendir cuentas, de responder a la ciudadanía por las políticas y programas que se comprometen. Y creo que esto, por ejemplo, es algo que puede haber estado ocurriendo 
Entrevista con la chilena Alejandra Faúndez: "En el caso de América Latina, el sofisticar la evaluación no tiene que ver con sofisticar el método. Tiene que ver con integrar la complejidad de esta región al análisis, que son caminos distintos"

en Brasil, por ejemplo, donde mucha gente del mundo de la comunidad, de la Red Brasileña de Evaluación, se haya resistido de no hacer ningún aporte al gobierno actual, con fuertes razones, sin duda. Sin embargo, eso necesariamente va a producir una brecha ahí, una brecha que después van a tener que volver a transitar. Ahí me parece que hay un riesgo más que un beneficio. Esa interfaz de articulación entre lo público y la comunidad de evaluación tiene que mejorar en todos lados, desde la autonomía y la independencia.

Sobre los desafíos, también me parece que tienen que ver con cuestiones técnicas. Hay desafíos metodológicos como integrar cosas no presenciales, trabajar mejor las dinámicas virtuales, no sobreexponer o sobresaturar a determinadas comunidades o actores en el campo de las entrevistas, en fin, ese tipo de cuestiones que yo creo que hay que revisarlas y hay que estar permanentemente revisándolas. También pienso que hay espacios de articulación que no debiéramos perder, por ejemplo, esto de las redes nacionales y regionales de evaluación.

Ya en el campo de género y derechos humanos, a mí me parece que tiene que haber capacidad de seguir cuestionando todo. Julieta Kirkwood -una feminista chilena que ha sido maestra de generaciones y que trabajó en FLACSO Chile- siempre dijo que había que sospechar de absolutamente todo y ponerlo bajo la lupa del género, esa es una gran lección para la evaluación.

También pienso en las generaciones de recambio. O sea, yo ya llevo no sé, 20, 30 años en estos temas y me encantaría que hubiese mujeres jóvenes de las nuevas generaciones haciéndose cargo, dándole nuevos impulsos a estos temas, y estoy segura que las hay. No sólo por una cuestión de que es "políticamente correcto" que haya alternancia en la coordinación como algo deseable -para las organizaciones como la ReLAC, pero también en los gobiernos y las ONG-, sino porque también a mí me parece que las nuevas voces de evaluadoras/es vienen cargadas no solo de entusiasmo, sino también de saberes, de sus propias lecturas de estos contextos tan dinámicos, de cosas que a lo mejor uno se está perdiendo de conocer, visibilizar, valorar y demostrar si no hay espacio para ellas/ellos. Pienso que puede haber un acompañamiento, un intercambio intergeneracional formal incluso, que puede ser muy productivo y nutritivo para varias generaciones, y que además puede ser planificado, integrando gente nueva que se vaya haciendo cargo y asumiendo nuevas responsabilidades rescatando los aprendizajes de quienes les precedieron. Porque creo que estos cambios siempre refrescan los saberes y, al mismo tiempo, se valora la acumulación de experiencias diversas de vida, de trayectoria, lo que es siempre más positivo que negativo.

\section{¿Qué podríamos pensar para la enseñanza ampliada de la evaluación que abarque la diversidad del campo y el enfoque de género en sus diversas capas?}

Algunas cosas que pueden ir en esa dirección: una es el decálogo de evaluación con enfoque de género que elaboramos en EVALGender+ hace años atrás. Y eso se tradujo en un documento, en un mini video que puede ser una señal de hacia donde podrían apuntar las evaluaciones con enfoque de género, son directrices amplias y de muy fácil comprensión también. Después, lo segundo, puede ser una guía de guías. Me parece que algo así es un ejercicio súper sencillo de compilar cosas que a todos nos importa, y que son producción de conocimiento de mucha gente, de muchos lugares. Creo que eso puede ser súper interesante de seguir ampliándolo y mejorándolo, hicimos una hace unos años, que hemos estado actualizando cada dos años, eso puede aportar en la región desde nosotros mismos.

Respecto del género y la evaluación propiamente, nosotros hicimos tres guías que también pueden ser útiles (aunque pueden ser revisadas porque son más antiguas). Una es “Ampliando la mirada" fue un trabajo conjunto con las Oficinas regionales de UNICEF, del UNFOA, el PNUD y ONU Mujeres; otra la hicimos en conjunto con ONU Mujeres y CLEAR América Latina, y una guía específica sobre género y evaluación para el gobierno de Colombia hace unos pocos años atrás. 
Entrevista con la chilena Alejandra Faúndez: "En el caso de América Latina, el sofisticar la evaluación no tiene que ver con sofisticar el método. Tiene que ver con integrar la complejidad de esta región al análisis, que son caminos distintos"

\section{¿Te gustaría hacer alguna consideración final?}

Sigo insistiendo en la invitación a que nuevos evaluadores y nuevas evaluadoras se integren al grupo de género y derechos humanos de ReLAC, que podamos seguir ahí discutiendo, profundizando, generando conocimiento, materiales, compartiendo información desde una perspectiva más del crecimiento de esta comunidad. Creo que debemos generar instancias de colaboración y de aprendizaje entre nosotras, abrir la mente también para observar que uno puede estar haciendo las cosas con todo el rigor del mundo, pero a veces sin la posibilidad de mirar las fisuras que hay en el mar de rigideces que el patriarcado nos impone a todo el mundo. $Y$ a ver si integramos personas de Brasil a este grupo de género. Ahora mismo estamos impulsando nuevos liderazgos e iniciativas, Claudia Olavarría está asumiendo la nueva coordinación del grupo de género y es muy importante revisitar lo que hemos construido junto con aprovechar los cambios producto de la crisis pandémica, que son siempre una fuente de poderosos aprendizajes.

\section{Fuente de financiamiento}

No hay.

\section{Conflicto de intereses}

No hay. 